Referencia para citar este artículo: Krauskopf-Roger, D. (2019). Relaciones intergeneracionales, emancipación e independencia de jóvenes estudiantes chilenos. Revista Latinoamericana de Ciencias Sociales, Niñez y Juventud, 17(1), 75-87. doi:https://dx.doi.org/10.11600/1692715x.17104

\title{
Relaciones intergeneracionales, emancipación e independencia de jóvenes estudiantes chilenos"
}

\author{
DinA KRAUSKOPF-ROGER ** \\ Profesora Universidad del Desarrollo, Chile.
}

\begin{abstract}
Artículo recibido en marzo 3 de 2016; artículo aceptado en abril 19 de 2018 (Eds.)
\end{abstract}
- Resumen (analítico): en este artículo analítico se destacan transformaciones contemporáneas en las relaciones intergeneracionales, así como la emancipación e independencia dejóvenes estudiantes chilenos pertenecientes al último año de la educación secundaria y al último año de la educación superior. La metodología se basó en grupos conversacionales provenientes de varias regiones del país. Las relaciones con los modelos adultos tienden a ser cofigurativas, entre las cuales se destaca la contrarreferencia, una visión sobre la generación adulta más analítica, a la vez que en proceso de transformación. La impredecibilidad y la incertidumbre conducen a procesos de emancipación e independencia que pueden ser reversibles y asincrónicos. Los tiempos históricos, la polarización social, la pertenencia al nivel secundario o la formación superior introducen importantes diferencias en las perspectivas del curso de vida de los jóvenes.

Palabras clave: juventud, brecha generacional, transformación social, relación padres e hijos, sociedad contemporánea (Tesauro de Ciencias Sociales de la Unesco).

\section{Intergenerational relationships, emancipation and independence of Chilean students}

- Abstract (analytical): This analytical paper highlights the contemporary transformations in intergenerational relationships, emancipation and independence of Chilean youth who are in their last year of secondary education or the final year of higher education. The methodology involved holding conversation groups in different regions in the country. Relationships with adults tend to be co-figurative and are seen as counter-reference point. Their view of the adult generation is more analytical and, at the same time, in the process of transformation. Unpredictability and uncertainty lead to processes of emancipation and independence that can be reversible and asynchronous. The current moment in history, social polarization and being either a secondary or higher education student are factors that create major differences in the perspectives of how these young people believe their lives will develop.

Key words: Youth, generation gap, social transformation, parents-children relationship, contemporary society (Unesco Social Science Thesaurus).

\footnotetext{
* Este artículo de reflexión está basado en los resultados del proyecto Juventudes SOC 1108 denominado Transformaciones socioeconómicas, sociopolíticas y socioculturales de las y los jóvenes en el Chile contemporáneo, perteneciente al Anillo de Juventud auspiciado por Conicyt,Chile, coordinado por la Universidad de Chile con la participación de siete Universidades y centros de investigación. La investigación fue desarrollada entre enero de 2013 y agosto de 2014. Área: Sociología; subárea: Temas sociales.

** Profesora adjunta de la Universidad del Desarrollo, Chile. Co-investigadora Proyecto Anillo Juventud. Profesora Emérita de la Universidad de Costa Rica. Correo electrónico: dina.krauskopf@gmail.com
} 


\section{Relações entre as gerações, a emancipação e independência da juventude chilena contemporânea}

- Resumo (analítico): este artigo analítico destaca transformações contemporâneas nas relações inter-geracionais, a emancipação e independência dos jovens estudantes chilenos no último ano de educação secundária e no último ano de educação superior. A metodologia basearam-se em grupos conversacionais correspondentes a diversas regiões do país. As relações com os modelos adultos tendem a ser co-figurativas e são vistas como contra-referentes, uma visão sobre a geração adulta mais analítica e, ao mesmo tempo, em processo de transformação. A impredizibilidade e a incerteza conduzem a processos de emancipação e independência que podem ser reversíveis e assincrônicos. Os tempos históricos, a polarização social, ser parte do nível secundário ou de formação superior introduzem importantes diferenças nas perspectivas do curso de vida dos jovens.

Palavras chave: juventude, brecha geracional, transformação social, relações pais e filos, sociedades contemporânea (Thesaurus de Ciências Sociais da Unesco).

-1. Introducción. -2. El curso de vida. -3. Los procesos que acompañan el desprendimiento juvenil de la familia. -4. Los ritmos generacionales. -5. Aproximación metodológica. -6. Los modelos adultos como referentes identitarios. -7. Las figuras adultas como contrarreferencias. -8. Las relaciones generacionales cofigurativas. -9. La independencia y la emancipación juvenil. -10. Conclusiones. -Lista de referencias.

\section{Introducción}

El paso del siglo XX al XXI estuvo marcado por cambios vertiginosos, tanto para las sociedades como para las juventudes. Las recientes transformaciones sociales, económicas y culturales en Chile, Latinoamérica y el mundo han impactado directamente las representaciones y las identidades con las cuales se estructuran los grupos sociales juveniles, por lo que es necesario avanzar en el conocimiento sistemático de los modos como las personas jóvenes se despliegan en las nuevas condiciones estructurales y subjetivas.

En ese contexto, el proyecto «Juventudes. Transformaciones socioeconómicas, sociopolíticas y socioculturales de las y los jóvenes en el Chile contemporáneo» se propone perfilar nuevas formas de comprensión de las condiciones juveniles en la sociedad chilena y su presencia como metáfora de la sociedad. La investigación — de la cual formamos parte- se desarrolló con una estrategia multimétodo que conjuga análisis de bibliografía producida, análisis cuantitativo de información secundaria y análisis cualitativo de información primaria. El estudio y la reflexión que presentamos se basan en el análisis cualitativo referido a los procesos de independencia o sujeción en las relaciones intergeneracionales.

Las personas jóvenes requieren reconfigurar las relaciones intergeneracionales para que sus sentimientos de adecuación y seguridad provengan de sus propias realizaciones (Krauskopf-Roger, 2013), lo que en las culturas modernas se da en contextos que se transforman. Así, las estrategias derivadas del paradigma del futuro esperable (encarnado, supuestamente, en la adultez), como enfoque orientador de la preparación de la juventud, ven reducida su efectividad ante la falta de predecibilidad que se desprende de la interacción rápida de factores a escala mundial. A la vez, las familias han experimentado un debilitamiento del patriarcado y es notable el desarrollo de nuevas estructuras en su funcionamiento (Arriagada, 2007; Olavarría, 2014).

Presentamos el análisis de los resultados obtenidos a partir de los grupos conversacionales del proyecto Juventudes del Anillo de Investigación en Ciencias Sociales-Conicyt denominado «Transformaciones socioeconómicas, sociopolíticas y socioculturales de las y los jóvenes en el Chile contemporáneo», el cual definió una metodología no directiva para conocer la subjetividad juvenil ${ }^{1}$.

1 Memoria del III Seminario Internacional Proyecto Juventudes SOC 1108. 
En nuestro trabajo se abordaron las características emergentes en las relaciones intergeneracionales, principalmente con las figuras parentales. Para su comprensión, relevamos los marcos conceptuales que se presentan a continuación, referidos al enfoque de curso de vida, las nociones concernientes a los procesos que dan lugar al desprendimiento de la familia, los ritmos en las transformaciones intergeneracionales y la implementación de directrices propias de vida.

\section{El curso de vida}

A mediados del siglo XX se replantean las perspectivas dominantes que le atribuían al desarrollo humano una secuencia de estadios con carácter unidireccional e irreversible (Lombardo \& Krzemien, 2008). En contraste con el uso extendido del concepto de ciclo de vida, el cual ha dado lugar a programas y políticas que se basan en la división en etapas de la vida humana y en la edad, Elder demuestra en sus investigaciones que los acontecimientos y roles no siguen necesariamente una secuencia dada, pues el curso de vida refleja la intersección de factores históricos y sociales con la biografía personal (Elder, 1985).

En el enfoque de curso de vida se enfatizan la interdependencia, los lazos sociales y la interconexión relacional de las experiencias sociales e individuales en varios niveles, vinculados a través de las familias y las redes de relaciones compartidas (Elder, 1998). Se reconoce, además, que el comportamiento y las decisiones no ocurren en un vacío y que existen circunstancias identitarias de localización geográfica y sociohistórica que afectan de modo diferenciado las historias de vida. En la variabilidad y heterogeneidad de los cursos de vida se encuentran presentes dimensiones como el sexo, la clase social, la estructura familiar, la etnicidad, la religión, y los apoyos derivados del capital cultural y económico; por lo tanto, el comportamiento y las decisiones no ocurren en un vacío.

Al respecto, Caffe (2015) destaca que las experiencias tienen un impacto en las transiciones y eventos en el mismo individuo, influyen en resultados posteriores con acumulaciones de desventajas o ventajas, afectan el curso de la vida de las personas vinculadas y los patrones intergeneracionales. Las transiciones resultan, por lo general, en cambios de estatus, identidad social e involucramiento en roles, y es posible que se reviertan o sean contratransiciones. Se pueden distinguir puntos de inflexión (turning points), periodos críticos y sensibles. Estos son los momentos en que se producen cambios significativos (biológicos, sociales, ambientales) y pueden causar efectos en el curso de la vida (Caffe, 2015). En este trabajo analizamos la salida de la educación secundaria y la salida de la educación superior como puntos de inflexión.

\section{Los procesos que acompañan el desprendimiento juvenil de la familia}

En el curso de la vida, la fase juvenil va acompañada de desafíos cruciales, vinculados al desprendimiento de la familia y la ubicación social. Los procesos de emancipación e independencia, la individualización y el desarrollo de la capacidad de agencia forman parte del repertorio con el cual esto se instrumenta. La emancipación de la gente joven se concreta en la separación del hogar con idoneidad para enfrentar personalmente los acontecimientos y acceder a la independencia de la intervención familiar. La independencia juvenil se relaciona, por lo tanto, con la competencia para actuar, hacer y elegir sin intervención o tutela ajena. Para Sen (2000), la libertad de agencia es la capacidad de uno mismo para potenciar las metas que desea. Hacerse cargo de la vida propia no es el resultado de la libre voluntad ni lo determinan las estructuras, sino que su carácter disposicional se desarrolla en la complejidad objetivo-subjetiva del habitus (Capdevielle, 2011).

La demanda contemporánea de individualización responde a un modelo cultural identitario fundado en los principios de independencia, flexibilidad y autorrealización individuales que se dan, en gran medida involuntariamente, en una fase de búsqueda y de ensayos históricamente prescritos (Beck \& Beck-Gernsheim, 2001). Esto incide en la dirección de la independencia, pues 
... por un lado, llegan la libertad y la decisión; por el otro, la obligación y la realización de las exigencias internalizadas del mercado. [...] Eso significa también que la individualización no es lo mismo que la individuación ${ }^{2}$, más bien se trata de un híbrido compuesto de conciencia de consumo y de autoconciencia (Beck \& Beck-Gernsheim, 2001, pp. 22-68).

\section{Los ritmos generacionales}

La fase juvenil ocupa, en el curso vital, un número creciente de años; la pubertad se da a edades más tempranas y la vida se prolonga en el contexto de la velocidad de los cambios. Esto incide en la redefinición de los límites y fronteras de las edades; las distancias generacionales de los jóvenes con los adultos se modifican y resignifican. Toman relevancia la rápida obsolescencia de conocimientos y técnicas, las expresiones culturales y los ámbitos de conocimiento propios de las nuevas generaciones, y los derechos y la equidad de género (Krauskopf-Roger, 2010), lo cual es claramente percibido por la gente joven. Al respecto, Leccardi (2014) señala que «... la mayoría de los jóvenes compara el propio tiempo de vida con el de sus padres... Es una capacidad inesperada de coger el espesor de los cambios intergeneracionales y su incidencia en las construcciones biográficas» (p. 152).

En relación con esto, Margaret Mead, en un valioso texto de 1971, anuncia las transformaciones generacionales y distingue tres tipos de culturas: 1) la cultura posfigurativa, en la que el cambio es tan lento e imperceptible que el pasado de los adultos es el futuro de cada nueva generación y esta aprende fundamentalmente de sus mayores, tradición que está diluyéndose ante la velocidad de los cambios. 2) La cultura cofigurativa aparece en las civilizaciones que han desarrollado técnicas para la incorporación del cambio y parece ser una fuerte tendencia de nuestros tiempos; los jóvenes recurren generalmente a alguna forma de aprendizaje a partir de los pares, los compañeros de juego, los condiscípulos. 3) Mead señala que en la contemporaneidad ingresamos en un periodo sin precedentes en la historia, con una cultura prefigurativa en la que los jóvenes asumen un nuevo estatus mediante la captación del futuro desconocido; los adultos aprenden de estos.

Como plantea Leccardi (2014, p. 57), «en la primera modernidad, el recambio entre las generaciones constituía la medida y el parámetro de referencia de los ritmos de mutación». Actualmente, las generaciones mayores afrontan un horizonte de vida más prolongado en un marco de veloces cambios, y experimentan situaciones sin precedentes, que demandan otras perspectivas en su recorrido de vida y en las relaciones con las nuevas generaciones. En dicho contexto, marcado por tantos cambios, el relevo generacional deja de ser la mejor medida.

\section{Aproximación metodológica}

En el proyecto Juventud se diseñaron grupos de discusión que se desarrollaron con técnicas conversacionales. La información se genera a partir de la discusión, en el supuesto de que la vida social es una conversación. Los informantes tienen derecho a hablar y participar a través de su punto de vista, lo que da lugar a una conversación grupal (Cano, 2008).

Se reunieron grupos de jóvenes con características similares, a los que se estimuló para que plantearan libremente cuáles eran los aspectos más importantes del momento por el que pasaban. Los propios investigadores, en el rol de moderador y observador, condujeron los grupos. En materia de cursos de vida, se apuntó a dos momentos críticos que incitan a la toma de conciencia para las acciones o decisiones en el curso de vida: salida del último año de la educación secundaria y del último año de la educación superior ${ }^{3}$.

2 La individuación es entendida, en la psicología del desarrollo, como el proceso que lleva a la diferenciación requerida para la elaboración de identidades. Se acentúa en la fase inicial de la adolescencia con oposición, esto es, conductas contrarias a lo esperado por las figuras parentales en procura de otras adquisiciones identitarias y visiones de mundo.

3 En la educación secundaria de Chile se encuentra casi la totalidad de la población correspondiente. 
En la muestra utilizada en la investigación se consideraron 22 grupos, con un promedio de diez estudiantes por grupo, en su gran mayoría en igual proporción de hombres y mujeres. Estos pertenecían a liceos públicos comunes, colegios particulares subvencionados, privados, laicos y católicos, y establecimientos de educación superior, tanto pública como privada, de varias regiones del país, particularmente aquellas a las que pertenecen los investigadores del Anillo y que abarcan desde el valle central hasta el sur austral ${ }^{4}$. La recolección de la información se realizó en el año 2014 y el equipo investigador ha analizado los resultados temáticamente. Resta conocer cuáles son los momentos críticos en el curso de vida de las personas jóvenes que no logran ingresar a la educación superior o pertenecen a grupos específicos, como etnias o comunidades en aislamiento rural.

En el estudio que presentamos, de carácter exploratorio y flexible, se reflexiona sobre momentos de transición vinculados a una amplia gama de preocupaciones existenciales y sociales, entre las cuales privilegiamos los discursos emergentes con cierta hegemonía y mayor voz sobre las relaciones intergeneracionales, principalmente con las figuras parentales, las modalidades de relación, la emancipación, la independencia, la individualización y la agencia. Mediante el análisis de contenido procuramos obtener el punto de vista de los sujetos respecto de sí mismos y su realidad social, sus modos comunes o diferenciados, para hacer interpretaciones de segunda instancia y captar las configuraciones subjetivas que los jóvenes reconstruyen en la implementación de directrices propias de vida y las relaciones intergeneracionales. En el contexto actual de prolongación de la vida, los cambios acelerados, las modificaciones del recorrido existencial, la rápida obsolescencia de los instrumentos y la superación de conocimientos, resulta valioso obtener claves sobre las perspectivas con las cuales las personas jóvenes lidian con su camino personal y social, así como el manejo de relaciones y referencias identitarias (Krauskopf-Roger, 2010). Debido a ello, justamente, nuestro análisis comienza con las figuras parentales.

\section{Los modelos adultos como referentes identitarios}

Las figuras parentales actúan como modelos, pero perciben confusos los tiempos actuales, lo que facilita la exacerbación de un control adultocéntrico, en particular sobre los estudiantes de secundaria de colegios públicos. Se mantienen características de épocas pasadas, en las cuales los adultos son importantes referentes identitarios por su experiencia, poder y guía para la conformación de la trayectoria juvenil. Las conversaciones sugieren, por parte de los padres, una mayor demanda de logros exitosos en las áreas en las que ellos no los tuvieron; dicha demanda puede estar acompañada de preocupación, en el sentido de que los jóvenes no cometan sus mismos errores.

Los esfuerzos de los padres para que sus hijos puedan cumplir con las expectativas se constituyen en una alta presión, la cual se acentúa cuando estos últimos son la primera generación de universitarios en la familia.

Mujer. Universidad pública

... cuando estaba en $4 .^{\circ}$ medio y era el tema de que tú eres buena alumna, tiene que irte bien en la prueba, tienes que salir a los 5 años y al $6 .^{\circ}$ tienes que tener el auto y el $7 .^{\circ}$ un departamento y al $8 .^{\circ}$ estar casada y tener dos hijos, o sea no... quebrar ese esquema, digamos, de expectativas que generan tu familia, tus papás, tus abuelos, la gente que te rodea; igual es complicado, sobre todo cuando uno es de los primeros profesionales que salen de la familia.

Mujer. Educación secundaria pública

La juventud pasada era como más reservada, [...] y la de ahora es como más liberal, más loca. Como que cosas que no se cometían antes, ahora es como la gran mayoría... Entonces por eso siempre los papás como que ahora nos tratan de cuidar más, para que no cometamos errores que antes eran muy rígidos.

4 Se trata de la Región Metropolitana, la Quinta, Sexta, Séptima, Octava y Décima región. 
Hombre. Educación secundaria pública

Es como un martirio porque ellos no están viviendo lo mismo que estamos viviendo nosotros, entonces como que es difícil conectarse...

La importancia tradicional del referente adulto puede perturbar la transición de la salida del colegio, por demandar una trayectoria predeterminada para ser «alguien» (paradójicamente, poco real y definido). El peso de este tipo de tensiones afecta, en los estudiantes secundarios, el desarrollo de elaboraciones identitarias; además, genera inseguridad en los procesos de individualización y agencia para poder abrirse a nuevas decisiones y alternativas de elecciones. Para los jóvenes de establecimientos de educación secundaria pertenecientes a los estratos sociales con mayores recursos, las expectativas apuntan a la conservación o superación de los niveles de éxito profesional de los padres (posgrados y otros).

Mujer. Educación secundaria pública

Yo creo que igual hay temor, temor de no poder, de tomar la decisión errónea, como si me voy a equivocar de carrera, si es lo correcto lo que estoy haciendo, y eso igual es porque muchas personas te presionan a que tú tienes que ser un profesional, pero muchas veces no haces lo que tú quieres, pero solamente te ponen la meta de que tú tienes que ser alguien.

Las fuertes tensiones que producen estas relaciones no están presentes cuando las figuras adultas ya no son experimentadas como factores sociales e identitarios predeterminantes, como veremos a continuación.

\section{Las figuras adultas como contrarreferencia}

A partir del análisis hecho, sobresale la aparición de figuras parentales cuyas características como modelos me han llevado a llamarlas contrarreferentes. En estos casos se ha debilitado la_visión de la generación anterior como referente al cual seguir y ha pasado a ser cuestionada para orientar a las personas jóvenes. Ciertas experiencias de los padres son valoradas como lo que no es conveniente seguir, o sea, son útiles como referencia disuasiva. Esto es particularmente claro en las mujeres. Se han modificado los roles de género y las muchachas procuran afirmar sus propios recursos de autonomía productiva y económica antes de contraer matrimonio. Las hijas, aun cuando la maternidad implica una gratificación marcada, rechazan la postergación personal que entraña el modelo materno.

Mujer. Universidad pública

Mi mamá se dedicó a los hijos, a nosotros, y se postergó mucho [...] Es muy feliz siendo mamá, porque mi mamá es profesora... Nunca ejerció tanto tiempo y eso es lo que yo antes no entendía... Creo que la vida de ella, a la de sus hijos, es superdistinta, porque nosotras... Bueno, yo sí quiero tener hijos, pero en un largo plazo también y tampoco me quiero postergar por ellos.

En esa línea, los estudiantes y las estudiantes manifiestan tener conciencia de la reducida amplitud de opciones y el impacto de los acontecimientos históricos que están en la base de las construcciones biográficas de los padres en relación con las propias, coincidiendo con lo encontrado por Leccardi (2014).

Varón. Educación secundaria pública

... El golpe de Estado... Sobre todo, por ejemplo, la juventud de antes, los papás eran demasiado, eran como mili... como los milicos... Era como una represión y ahora el joven de ahora es liberal...

El modelaje parental con efectos disuasivos, que hemos denominado contrarreferencia, ha sido un hallazgo del presente análisis. Esta perspectiva va más allá, en especial cuando los jóvenes observan que en las figuras parentales aparecen replanteamientos sobre los criterios que guiaron sus propias biografías, con la consiguiente apertura a la elaboración de otras directrices en la conducción de momentos críticos del propio curso de vida. Esto da lugar a las relaciones cofigurativas que analizamos a continuación. 


\section{Las relaciones generacionales cofigurativas}

A las personas adultas se las puede ver como figuras de acompañamiento, apoyo, asesoría e interlocución, alcanzando una relación intergeneracional expresada con satisfacción por las personas jóvenes. Es notable la existencia de figuras parentales que dejan de imponer el modelo que vivieron, se abren a las nuevas perspectivas de sus hijos e hijas, los apoyan y respetan, y fomentan la agencia.

Varón. Universidad pública

Ella siempre ha dicho que sea libre, cualquier cuestión me dice: "Vivelo y siempre tú enfocado, sí, en no perderte; intenta no perderte, pero vívelo». Porque yo creo que es lo mismo, porque ella no lo vivió, se postergó mucho en su vida para sus hijos.

En lo político, se encuentran padres que fueron militantes con una óptica tradicional que incidía históricamente en su experiencia de juventud; los estudiantes y las estudiantes observan que la velocidad del cambio transforma los propios cursos de vida de la generación adulta. En ese sentido, ellos y ellas perciben el accionar político y social de la juventud más diversificado, no despolitizado, con una interacción generacional cofigurativa.

Mujer. Universidad privada

... había una idea de hacer familia, porque había una idea también de ser país... Creo mucho en la participación juvenil dentro de los espacios en todo ámbito; el movimiento estudiantil... alzó esta temática de la educación como prioritaria ... pero ahora es como normal y todos lo hablan, desde los secundarios hasta grandes, hasta mis abuelos están hablando de esto y no se imaginaron cambiar nada de la sociedad...

Por otro lado, cabe destacar la valoración de la diversidad, lo que incluye la diferencia generacional en las interacciones. Para algunas personas jóvenes, estar dispuesto a escuchar y pensar frente a alguien radicalmente distinto permite generar conversaciones productivas, aun cuando se hagan en contextos de tensión. Además, como consideran importante la transmisión generacional de conocimientos, existe aprecio por las conversaciones. Esto se observa en grupos de jóvenes con más oportunidades de desarrollar visiones variadas y capacidad de agencia.

Mujer. Universidad privada

Yo creo que como que nosotros estamos buscando la diversidad en general; yo, por lo menos, siento que busco la diversidad... Es como rico hallar espacios de encuentro quizás no solo con jóvenes, así como con gente de todas las edades.

Además, si bien no se manifiestan tendencias prefigurativas, se vislumbra la aparición de jóvenes que señalan razones para llegar a convertirse en referentes prefigurativos de las generaciones mayores. Varón. Educación secundaria

Lo malo que tenemos los jóvenes es que queremos inculcarles a nuestros papás lo nuevo de ahora, siendo que los papás no tienen la mente como pa procesar todo lo que hay ahora...

Finalmente, cabe señalar que la retribución intergeneracional es parte de las preocupaciones de las personas jóvenes de todos los sectores socioeconómicos, ante las decisiones respecto a su curso de vida.

Varón. Educación secundaria privada subvencionada

Por lo menos, yo también quiero jugármela por mi futuro, y también para devolverle la mano a lo que están haciendo mis papás.

A continuación nos referiremos a las principales tensiones halladas en el desarrollo del curso de vida con la familia, en las que se expresaron la independencia, la emancipación y la distribución del tiempo familiar. Profundizaremos en las dos primeras, pues son aspectos claves en las relaciones intergeneracionales.

\section{La independencia y la emancipación juvenil}

Vale la pena señalar que la mayoría de los jóvenes estudiados tenían dependencia habitacional, lo que plantea la tensión protección-independencia. Se aprecia que la vida familiar durante la fase 
juvenil es clave; constituye un tejido cuyas características inciden en su bienestar y dirección de curso de vida, particularmente durante el periodo adolescente, cuando asisten a la educación secundaria. Las personas jóvenes destacan la centralidad del apoyo, su incondicionalidad, el papel estabilizador de sus estados de ánimo y también, en algunos casos, el peso de ser demasiado importantes emocionalmente para las figuras parentales.

Para la familia, según las estudiantes y los estudiantes universitarios, la independencia involucra asumir responsabilidades. Se genera la paradoja de que la independencia se expresa en responsabilidades y que, a mayor número de responsabilidades, las personas jóvenes sienten que se diferencian menos de la adultez. Adicionalmente, los indicadores de responsabilidad pueden fluctuar desde aspectos mínimos, como avisar, hacerse cargo de errores o transgresiones, trabajar para sus gastos personales, hasta asuntos de mayor peso en materia de autonomía. Son variantes subjetivas en el autorreconocimiento de la independencia y de la importancia de la emancipación.

Varón. Universidad privada

Como que ellos ya tienen asumido, o sea, ya te criamos, ya erís grande, si te metes en un condoro tú vas a tener que asumir.

Varón. Universidad pública

La independencia también marca el punto en que erís capaz de administrarte por ti mismo... Mujer. Universidad privada

... igual, uno ya empieza a generar una independencia mental y que se nota con el entorno que uno empieza a marcar sus propios límites, pero ahora lo que nos falta es eso, es tener como el estado económico pa poder decir ahora yo me separo de ti, mamá, papá, y puedo generar mi propio entorno...

En estudiantes secundarios de colegios públicos, los logros están asociados a una mayor emancipación y responsabilidad intergeneracional, lo cual puede tener costos e involucrar frustraciones limitantes en las posibilidades de orientar su curso de vida hacia las metas más satisfactorias.

Varón. Educación secundaria

... tengo cómo costearme la educación, pero tampoco es la idea cargarle ese peso tan grande a mis padres, pues ellos no siempre van a estar acompañándome en este proceso; entonces, si yo no me muevo por sí solo, trato de aprender, cometiendo errores o teniendo soluciones, no voy a aprender cómo seguir avanzando después...

Mujer. Educación secundaria

... nosotros en este Liceo no tenemos el respaldo económico... como otros colegios ... Nuestra visión es como pa ser un poco más que nuestros papás, y como ellos no tienen un respaldo económico nosotros ehhm, tenemos que esforzarnos el doble pa y no podemos tampoco como darnos el lujo de elegir lo que queremos hacer, po, tenemos que elegir algo que nos dé algún capital económico pa darle un buen vivir también a las generaciones que vienen después de nosotros... Varón, mismo grupo... Y a las anteriores.

La independencia en los jóvenes actuales no es lineal, unívoca y sincrónica. El proceso tiene un plazo extenso. Difiere de los procesos de emancipación en contextos posfigurativos, en los que esta se produce por el alejamiento del hogar y da cuenta de una transformación en las relaciones con los adultos.

En ese orden de ideas, la permanencia en el hogar parece cambiar de signo, particularmente en relación con tiempos posfigurativos. Irse de la casa hoy en día no siempre significa emancipación ni es una transición que no pueda revertirse, ya que es posible que se presente: 1) la emancipación aparente con mantención de elementos de dependencia; 2) la reversibilidad en las decisiones como el retorno a la protección del hogar, y 3) el desarrollo de agencia e independencia con permanencia en el hogar. Volver al hogar implica generalmente pérdida de autonomía, pero pueden encontrarse modalidades de retorno con una especie de asociación intergeneracional protectora que contribuyen a la independencia, en la medida en que aportan condiciones para un mejor desarrollo de las bases económicas. Con frecuencia se destaca que la permanencia ofrece la ventaja de permitir la acumulación de capital económico que sustente la agencia. 
Varón. Universidad privada

... y mucha gente da como erróneo "Ah, se fue de su casa, ya es demasiado independiente, demasiado maduro, pero en el fondo... Varón, mismo grupo: Pero podís seguir siendo un mamón igual.

Varón. Universidad pública

... viví solo... pero me agoté, asi que volví... Tu responsabilidad de que si no pagaste la luz te la van a cortar, entonces, porque siempre hay alguien, digamos, que la puede pagar... Hay cosas que igual tú echas de menos, de repente tonteras, pero no sé, el hecho de que no tienes que avisarle a nadie que vas a llegar más tarde, o no sé, que vas a llegar con alguien o cosas por el estilo.

Varón. Universidad privada

... si uno vuelve también a la casa de los papás, porque logra gestionar mejor las platas; entonces ahorras, digamos, no tienes que hacerte cargo, tan responsable, de algunas cuentas, podí irte a medias, podi apoyar.

Mujer. Universidad pública

... volví a vivir con mi mamá y ahora he vuelto a ver un proceso de que estamos vendiendo la casa, entonces ahora como tengo pensado de instalar este centro de yoga, pensé habilitar ahí mismo el segundo piso para mí...

Varón. Universidad privada

... las comodidades son impresionantes [risas]. En un futuro no muy lejano, tengo la idea de quedarme un tiempo, quizás poder juntar como plata y después mandarme a cambiar, no salir solo a la jungla. Además que mis papás son supertranquilos, no tengo problemas.

La comprensión generacional respecto al hecho de que las personas jóvenes quieran irse de la casa varía y algunas opinan que influye la edad en que lo hicieron los padres. La emancipación es fuente de tensiones, sobre todo en familias de corte más tradicional. Para las jóvenes mujeres existen visiones de género en algunas figuras parentales que no aceptan la separación del hogar, incluso esperan que la hija se vaya casada; es lo que llamo emancipación tradicional.

Varón. Universidad privada

... mi papá se fue a los 18 de su casa y mi mamá también. Ellos a los 18 nos dijeron «Sería bacán que acá en Chile existiera eso que a los 18 ustedes se fueran».

Mujer. Universidad privada

... son tan mayores y vivieron en otra generación, que les cuesta mucho más aceptar que yo a los 23 años puedo ser mucho más; mi mamá se fue de su casa a los 35, entonces no entienden que yo me quiero ir de la casa el próximo año, cuando esté trabajando.

Mujer. Universidad privada

... ella sabe que yo me voy el próximo año, o sea, si consigo pega me voy... pero la idea es que me vaya casada...

En relación con esto, Antonia Garcés (2014) destaca matices diferentes en el grado de autonomía y convicción de logro con que los jóvenes de los sectores populares y los de sectores altos viven la agencia y los procesos de individualización. En los primeros aparece el «sí se puede» y en los sectores altos se expresa como «todo esto depende de mí», pues la posición es de mayor confianza en sí mismos: «Si me fuera mal no sería tan dramático, me repongo; aun cuando perdí en algo, no voy a perder tanto». En el nivel universitario de las personas jóvenes participantes apreciamos la existencia de «jóvenes con mayores recursos reflexivos para interpretar la incerteza del futuro como multiplicación de posibilidades virtuales y la impredecibilidad que, hoy, se asocia al futuro como potencialidad adicional en lugar de como límite a la acción» (Leccardi, 2014, p. 131).

\section{Conclusiones}

A partir del análisis efectuado es posible concluir que, en el caso de Chile, un sector importante de jóvenes encarna nuevas formas al abordar las relaciones intergeneracionales, los procesos de 
independencia e individualización. Las interpretaciones que aquí se presentan pueden someterse a nuevas y amplias investigaciones que vayan más allá de los esquemas habituales, para profundizar en la complejidad de las implicaciones económicas y relacionales, además de sistematizar las estrategias emergentes en momentos transicionales del curso de vida.

En síntesis, a partir de las conversaciones grupales efectuadas consideramos que los aspectos más destacados que se observan son:

a) Culminación de los estudios: la inserción social futurizada_

En las conversaciones sostenidas queda en evidencia que ambos pasos — la culminación de la educación secundaria y el término de la educación superior- constituyen puntos de inflexión en el curso de vida, en los cuales los jóvenes están sujetos a realizar definiciones en momentos institucionalmente predeterminados (Canales, 2015). Estos momentos son vividos de modo crítico y muy sensible, ya que ponen en tensión las condiciones y adquisiciones juveniles frente a la demanda de organizar respuestas más allá del fortalecimiento identitario. Es un presente que se futuriza en el encuentro de un sitio propio diferenciado, en mayor o menor grado, de la familia.

Cabe señalar que son diversas las exigencias que predominan en cada nivel educativo (secundario y superior), así como las opciones que se vislumbran. Las condiciones socioeconómicas y de género, al igual que las dificultades para la incorporación al mercado laboral, constituyen una fuerte preocupación en estudiantes de educación secundaria pública. La disolución paulatina del Estado de bienestar y la ausencia de implementación del Estado social se hacen más notorias cuando las demandas de incorporación social son más inciertas.

b) Las transformaciones de la emancipación e independencia del hogar

Prácticamente, todas las personas jóvenes de la muestra analizada se encuentran viviendo en las casas de sus padres. La actitud incondicional de las figuras parentales para la permanencia de los hijos e hijas mayores en el hogar posiblemente responda a la gran valoración emocional que tienen actualmente. Al respecto, Beck y Beck-Gernsheim (2001) destacan la importancia central que toman los hijos e hijas para las parejas contemporáneas, pues la individualización afecta seriamente la permanencia del amor, con posibilidades de disolución: «Lo que queda es el hijo... como último garante de la duración, como arraigo de la propia vida» (p. 106).

Las condiciones de vida y los patrones de las personas cercanas son fundamentales en lo referente a la respuesta al estrés, la vulnerabilidad y la promoción de comportamientos resolutivos. Esto implica tensiones y conflictos, especialmente cuando las metas individuales difieren de las necesidades de la familia como unidad colectiva. Para una mayoría de estudiantes, tanto de colegios públicos como privados, es clave el peso de las expectativas y esfuerzos de la familia; dichas expectativas pueden representar presiones objetivas y subjetivas en la responsabilidad juvenil de asumir exitosamente sus trayectorias de avance social e incluso en cuanto a retribuir lo recibido.

La emancipación juvenil difiere según el nivel económico y el género: sus prácticas y logros están vinculados a los procesos de desigualdad estructural y las características líquidas que introduce la modernidad en el contexto del habitus. En estas condiciones, los procesos de independización no buscan niveles absolutos, sino graduados de acuerdo con la conveniencia que ofrecen las circunstancias. En términos de género, persiste para algunas jóvenes la exigencia de la emancipación tradicional: irse casada. En las relaciones generacionales cofigurativas es característica y funcional la flexibilización de los plazos con secuencias de diversas autonomías. A mayor nivel de autonomía económica, mayores posibilidades de independización, pero menor urgencia de esta y viceversa.

Irse de la casa con capacidad de agencia es parte fundamental de la emancipación familiar, pero la expresión de la independencia no es unívoca ni lineal. Aunque se valora, no es prioridad irse de la casa; es prioritario encontrar trabajo. La separación del hogar no es siempre permanente: influyen en el retorno las dificultades para la autonomía, así como las posibilidades de desarrollar bases económicas.

En todos los grupos analizados se observan necesidades de individualización frente a las condiciones de una sociedad que no ofrece protección ni garantías para lograr la integración. La agencia está vinculada a la individualización, en cuanto permite la confrontación de las trayectorias 
predeterminadas por los modelos adultos y la reelaboración de las experiencias y los resultados. Los jóvenes se encuentran con un país que supone un alto proceso de individualización, a la vez que lo impide o dificulta.

Vale la pena destacar que el descubrimiento de expectativas propias puede llevar a algunos jóvenes a dejar de priorizar la universidad y experimentar un quiebre de las pretensiones introyectadas por las demandas del entorno adulto. Esto conlleva una nueva presión (interna y externa) por conseguir resultados acordes con sus decisiones y enfrenta a la persona joven a las dimensiones sociales, económicas y culturales de su entorno. Así es como la capacidad de agencia alcanza mayor valoración y centralidad para asumir las consecuencias de sus actos y articular los avances en la individualización.

c) Procesos de cambio en las generaciones

Los tiempos históricos influyen marcadamente en las relaciones generacionales chilenas. Leccardi (2014) señala que cada generación está forzada a confrontarse de modo constante con una pluralidad de procesos de cambio que la atraviesan, transformando los escenarios cotidianos. En general, las juventudes chilenas muestran nuevas perspectivas en sus relaciones con las generaciones mayores y reconocen tiempos biográficos diferentes, sobre todo en los grupos con mejores condiciones económicas. Se afirman, cada vez más, en sus identidades juveniles y los procesos de individualización, lo que se aprecia particularmente en estudiantes universitarios.

Los jóvenes con mejores condiciones para desarrollar su agencia consideran que los límites para llegar a la adultez son postergables y ambiguos, en tanto que la impredecibilidad no es necesariamente temida y más bien puede ser estimulante como campo de opciones. Se aprecia en los grupos estudiados que el ingreso al ámbito universitario abre más perspectivas.

La adultez, con sus presiones y responsabilidades, se vislumbra más cercana en las personas jóvenes de menor capital económico y cultural. La experiencia confrontada con predeterminación de opciones de difícil alcance no proporciona respuestas suficientes. La vivencia, más que de impredecibilidad, es entonces de mayor incertidumbre para lograr las metas que valoran, lo cual se da especialmente durante la educación secundaria. En este nivel, muchos de los mandatos sociales y las exigencias parentales y de otras figuras adultas son interiorizados, al tiempo que debilitan una visión propia para afrontar una pluralidad de situaciones. Por ello, debe estudiarse el enfrentamiento de las opciones contemporáneas de quienes no acceden a estudios técnicos y superiores para darle una importante consideración en las políticas públicas.

Las expectativas y presiones generacionales en los estudiantes y las estudiantes de colegios públicos muchas veces incluyen las demandas por superar lo conseguido por la familia. Sin embargo, en la medida en que pertenecen a un mejor estatus económico, las demandas que hacen los adultos apuntan a alcanzar el nivel de los logros familiares existentes, al igual que su renovación e innovación. Estas consideraciones, así como otras ya señaladas, contribuyen a evidenciar la desigualdad social del curso de vida y la necesidad de incluirlas en el diseño de políticas y programas sociales orientados a adolescentes y jóvenes.

d) Tensiones intergeneracionales y referentes identitarios

Los cambios sociales dieron lugar a nuevas tensiones en las personas adultas, entre estas la pérdida de eficiencia de los instrumentos tradicionales para orientar el desarrollo de sus hijos e hijas en el contexto de las demandas sociales contemporáneas. Las confrontaciones y conflictos responden, más bien, a cosmovisiones diferentes y no a la tradicional búsqueda de su poder en la lucha generacional, como se encontraba (y aún se encuentra) en las fases posfigurativas. Las figuras adultas más apegadas a las visiones tradicionales tienen dificultad para conceder validez a los planteamientos juveniles (esto amenazaría su autoridad y daría paso a la inseguridad), por lo que se pueden agudizar los abordajes adultocéntricos como forma de retención del poder adulto y también como medida de apoyo ante la falta de instrumentos innovadores frente a los cambios. Esto causa conflictos distintos, que trascienden la tradicional lucha generacional, los cuales pueden expresarse en bloqueos comunicacionales y discursos paralelos entre ambas generaciones. 
Existe claridad en lo referente a las diferencias generacionales, pero los énfasis entre jóvenes de secundaria (especialmente de menos recursos económicos) y jóvenes de estudios superiores son distintos. Parece que en los primeros predomina la visión de las figuras parentales como guía y referente identitario, y en los segundos hay más tendencia a verlos como contrarreferentes que producen disuasión y divergencia. Los hallazgos llevan a pensar que la contrarreferencia se produce a medida que el proceso juvenil avanza en el tiempo; esto posibilita la reflexión sobre los principios, prácticas y creencias que han guiado la conducción del curso de vida de las figuras parentales y los adultos significativos, contrastada con la vivencia juvenil de la contemporaneidad, puesto que consideran que tienen más oportunidades que la generación anterior.

Consideramos la contrarreferencia como una forma propia de la contemporaneidad en la elaboración de identidades y roles en la fase juvenil. A diferencia de los procesos de oposición e individuación del inicio de la fase juvenil, la contrarreferencia forma parte de los procesos de individualización; no busca la descalificación de la autoridad parental, dado que esta ya no reviste la certeza de tener todas las respuestas requeridas para enfrentar la rapidez de los cambios. Incluso la autoridad parental puede dar lugar a relaciones cofigurativas, casos en los que se modifica con nuevos elementos en la relación y comunicación intergeneracional; la valorización de la diversidad permite a varias generaciones tomar insumos para la elaboración de opciones y visiones que aportan a la diferenciación como parte del proceso de individualización. Esto está más restringido en figuras parentales de origen humilde y orientación tradicional.

La prolongación de la vida, la mayor variabilidad en la generación de proyectos acordes con circunstancias cambiantes y el reposicionamiento de las ventajas generacionales (innovación versus experiencia) dan nuevas perspectivas al curso de vida. Las experiencias y guías que las figuras adultas significativas recibieron durante su propio desarrollo, cuando son aplicadas a su descendencia en un horizonte diverso al correspondiente a su pasado, a menudo no se ajustan a las características e instrumentos del campo social que encuentran en la actualidad y les demandan otras perspectivas para apoyar el desarrollo de sus hijos en los momentos gravitantes de su vida. Estas evidencias son, para muchos padres y sus hijos e hijas, datos para nuevas comprensiones basadas en el reconocimiento de que los tiempos, al cambiar, necesitan otras estrategias, sin desdeñar aquellas que, a su juicio, siguen siendo claves o adecuadas para la directriz de vida hacia la cual apuntan.

Ya no se trata de una generación adulta preparada frente a una generación joven, carente de derechos y conocimientos que hay que preparar. Diferentes características y transformaciones en las interacciones estudiadas permiten contar con una visión más analítica de los cursos de vida juveniles, lo cual contribuye a perfilar nuevas formas de aprehensión de las condiciones juveniles y de las políticas requeridas en la sociedad chilena contemporánea.

\section{Lista de referencias}

Arriagada, I. (2007). Transformaciones familiares y políticas de bienestar en América Latina. En I. Arriagada (Coord.), Familias y políticas públicas en América Latina: una historia de desencuentros (pp. 125-149). Santiago: Cepal, UNFPA.

Beck, U., \& Beck-Gernsheim, E. (2001). El normal caos del amor. Las nuevas formas de la relación amorosa. Buenos Aires: Paidós.

Caffe, S. (2015, septiembre). Panorama del embarazo adolescente en Latinoamérica desde $l a$ perspectiva de curso de vida. Ponencia Foro Internacional para la Prevención del Embarazo Adolescente, Bogotá, Colombia.

Canales, M. (2015). Presentación de resultados. Memoria del III Seminario Internacional Proyecto Juventudes SOC 1108. Santiago de Chile: Universidad de Chile.

Cano, A. (2008). Técnicas conversacionales para la recogida de datos en investigación cualitativa: el grupo de discusión. Nure Investigación, 35, 1-4. Recuperado de: http://www.nureinvestigacion. es/OJS/index.php/nure/article/view/417 
Capdevielle, J. (2011). El concepto de habitus: «Con Bourdieu y contra Bourdieu». Revista Anduli, 10, 31-45. Recuperado de: https://dialnet.unirioja.es/descarga/articulo/3874067.pdf.

Elder, G. H. (1985). Life Course Dynamics. Ithaca: Cornell University Press.

Elder, G. H.. (1998). The life course as Developmental Theory. Child Development, 69(1), 1-12. doi:10.2307/1132065

Garcés, A. (2014). Memoria de la jornada de análisis Proyecto Juventud. Santiago de Chile: Universidad de Valparaíso.

Krauskopf-Roger, D. (2010). La condición juvenil contemporánea en la constitución identitaria. Última Década, 18(33), 27-42. Recuperado de: http://www.scielo.cl/scielo. php?script=sci_arttext\&pid=S0718-22362010000200003\&lng=es\&tlng=es. $\quad 10.4067 / \mathrm{S} 0718$ 22362010000200003.

Krauskopf-Roger, D. (2013). Adolescencia y educación. San José de Costa Rica: Euned.

Leccardi, C. (2014). Sociologías del tiempo. Santiago de Chile: Ediciones Universidad Finis Terrae.

Lombardo, E., \& Krzemien, D. (2008). La psicología del curso de vida en el marco de la psicología del desarrollo. Revista Argentina de Sociología, 6(10), 111-120.

Mead, M. (1971). Cultura y compromiso. Un estudio sobre la ruptura generacional. Buenos Aires: Granica.

Olavarría, J. (2014). Transformaciones de la familia conyugal en Chile en el periodo de la transición democrática (1990-2011). Polis, 13(37), 473-497. Recuperado de: https://scielo.conicyt.cl/pdf/ polis/v13n37/art25.pdf.

Sen, A. (2000). Desarrollo y libertad. Buenos Aires: Planeta. 